\title{
Association Between Renal Adverse Effects and Mortality in Patients With Hepatocellular Carcinoma Treated With Lenvatinib
}

\author{
YOSHINOSUKE SHIMAMURA ${ }^{1}$, KOKI ABE $^{1}$, TAKUTO MAEDA ${ }^{1}$, \\ TAKESHI MATSUI ${ }^{2}$, ATSUSHI ISHIGURO ${ }^{3}$ and HIDEKI TAKIZAWA ${ }^{1}$ \\ ${ }^{1}$ Department of Nephrology, Teine Keijinkai Medical Center, Sapporo, Japan; \\ ${ }^{2}$ Center for Gastroenterology, Teine Keijinkai Medical Center, Sapporo, Japan; \\ ${ }^{3}$ Department of Medical Oncology, Teine Keijinkai Medical Center, Sapporo, Japan
}

\begin{abstract}
Background/Aim: Lenvatinib, a multitargeted tyrosine kinase inhibitor, was recently approved for hepatocellular carcinoma (HCC) treatment in Japan; however, the association between proteinuria following lenvatinib administration in HCC patients and early mortality is unknown. This study aimed to examine the association between nephrotic-range proteinuria (NRP) and mortality and evaluated the risk factors for NRP among Japanese HCC patients treated with lenvatinib. Patients and Methods: We retrospectively analyzed 45 consecutive patients receiving lenvatinib from 2018-2019. Primary outcome was overall survival. Cox proportional hazards regression was used to evaluate the association between NRP and overall survival. Logistic regression analyses were used to identify NRP risk factors after lenvatinib initiation. Results: The median age was 66 years, 56\% were women, and $20 \%$ had pre-existing proteinuria. During a 1-year median follow-up, 24 died, and 5 developed NRP. Univariable logistic regression showed that pre-existing proteinuria was associated with higher NRP risk; however, the association was not significant after covariate adjustment. Following multivariable Cox analysis, NRP did not affect overall survival in advanced HCC patients receiving lenvatinib. Conclusion: Urinalysis findings should be monitored regularly in patients receiving lenvatinib because NRP incidence was comparable to that of prior
\end{abstract}

This article is freely accessible online.

Correspondence to: Yoshinosuke Shimamura MD, MPH, Department of Nephrology, Teine Keijinkai Medical Center, Sapporo, Hokkaido, Japan. Tel: +81 116818111, Fax: +81 116852998, e-mail: shimamura-yo@keijinkai.or.jp

Key Words: Hepatocellular carcinoma, lenvatinib, overall survival, proteinuria. studies. Identifying the predictors of NRP after lenvatinib initiation warrants further investigation.

Hepatocellular carcinoma (HCC) is one of the most common causes of cancer-related deaths worldwide (1). The treatment of HCC has dramatically changed with the introduction of molecular-targeted agents, such as lenvatinib and sorafenib (24). Lenvatinib, an oral multi-kinase inhibitor of vascular endothelial growth factor receptor 1-3, platelet-derived growth factor receptor $\alpha$, fibroblast growth factor receptor 1-4, and RET and KIT proto-oncogenes, demonstrated non-inferiority to sorafenib in terms of overall survival and progression-free survival among patients with advanced HCC, and it was approved in Japan for advanced HCC in March 2018 (4, 5). Common adverse effects associated with lenvatinib include diarrhea, fatigue, rash, and hand-foot syndrome, while nephrotic-range proteinuria (NRP) has also been reported in some patients $(4,6-9)$. Since most of the data regarding the incidence of NRP were derived from randomized clinical trials $(4,6)$, the association between NRP and overall survival among patients with advanced $\mathrm{HCC}$ receiving lenvatinib in a real-world setting is unknown. Therefore, we conducted a single-centre retrospective observational study to investigate the association between NRP after lenvatinib initiation and overall survival and identify the risk factors for NRP in Japanese patients with advanced HCC treated with lenvatinib. This study contributes new knowledge regarding the adverse effects of lenvatinib, which is used to improve the management of patients with advanced HCC.

\section{Patients and Methods}

Patients and study design. This was a single-centre, retrospective observational study based on clinical data collected at the Teine Keijinkai Medical Center, a 650-bed tertiary reference centre, in Sapporo, Hokkaido, Japan. We included consecutive patients with unresectable HCC diagnosed and staged according to the Liver Cancer 
in vivo $35: 1647-1653(2021)$

Table I. Baseline characteristics of the study participants according to nephrotic-range proteinuria.

\begin{tabular}{|c|c|c|c|c|}
\hline & All $(n=45)$ & Non-NRP $(n=40)$ & $\operatorname{NRP}(n=5)$ & $p$-Value \\
\hline Age, year [median $(25 \% 75 \%]$ & $66[55,72]$ & $66[55,72]$ & $69[66,73]$ & 0.24 \\
\hline Gender $(\%)$ & $25(56)$ & $22(55)$ & $3(60)$ & 1.00 \\
\hline BMI, $\mathrm{kg} / \mathrm{m}^{2}$ [median $\left.(25 \%, 75 \%)\right]$ & $23.1[21.5,25.0]$ & $23.1[21.5,25.0]$ & $21.6[20.3,25.0]$ & 0.59 \\
\hline Hypertension, $\mathrm{n}(\%)$ & $29(64)$ & $24(60)$ & $5(100)$ & 0.14 \\
\hline Diabetes mellitus, n (\%) & $14(31)$ & $13(33)$ & $1(20)$ & 1.00 \\
\hline Heart failure, $\mathrm{n}(\%)$ & $4(9)$ & $2(5)$ & $2(40)$ & 0.01 \\
\hline Chronic kidney disease, n (\%) & $18(40)$ & $16(40)$ & $2(40)$ & 1.00 \\
\hline ECOG-PS $(0,1, \geq 2)$ & 20 (44), 5 (12), 20 (44) & $17(42), 4(10), 19(48)$ & $3(60), 1(20), 1(20)$ & 0.32 \\
\hline Etiology, n (\%) (HBV, HCV, Others) & $4(9), 40(89), 1(2)$ & $3(8), 36(90), 1(2)$ & $1(20), 4(80), 0$ & 0.61 \\
\hline Macrovascular invasion, $\mathrm{n}(\%)$ & $23(51)$ & $21(53)$ & $2(40)$ & 0.60 \\
\hline ACE inhibitor, $\mathrm{n}(\%)$ & $6(13)$ & $5(13)$ & $1(20)$ & 0.53 \\
\hline $\mathrm{ARB}, \mathrm{n}(\%)$ & $21(47)$ & $18(45)$ & $3(60)$ & 0.65 \\
\hline NSAIDs, n $(\%)$ & $3(7)$ & $3(8)$ & 0 & 1.00 \\
\hline Prior treatment with sorafenib, $\mathrm{n}(\%)$ & $14(31)$ & $13(33)$ & $1(20)$ & 1.00 \\
\hline Transcatheter arterial chemoembolization, n (\%) & $27(60)$ & $23(58)$ & $4(80)$ & 0.63 \\
\hline Total bilirubin, $\mathrm{mg} / \mathrm{dl}[$ median $(25 \%, 75 \%)]$ & $0.7[0.5,1.0]$ & $0.7[0.5,1.0]$ & $0.5[0.4,1.0]$ & 0.46 \\
\hline Serum albumin, g/dl [median $(25 \%, 75 \%)$ ] & $3.8[3.4,4.1]$ & $3.9[3.4,4.1]$ & $3.7[3.6,4.0]$ & 0.64 \\
\hline Prothrombin time, $\%$ [median $(25 \%, 75 \%)$ ] & $87.5[81.4,96.3]$ & $85.3[78.7,95.7]$ & $97.9[91.4,98.3]$ & 0.11 \\
\hline Baseline AFP, IU/ml [median $(25 \%, 75 \%)]$ & $120[8.3,356]$ & $88[8.4,223]$ & $172[6.4,847]$ & 0.42 \\
\hline Serum creatinine, mg/dl $[$ median $(25,75 \%)]$ & $0.78[0.65,0.96]$ & $0.77[0.64,0.95]$ & $0.93[0.77,1.20]$ & 0.02 \\
\hline $\mathrm{eGFR}, \mathrm{ml} / \mathrm{min} / 1.73 \mathrm{~m}^{2}$ [median $\left.(25 \%, 75 \%)\right]$ & $66[52,83]$ & $68[52,85]$ & $62[46,76]$ & 0.15 \\
\hline Pre-existing proteinuria, $\mathrm{n}(\%)$ & $9(20)$ & $6(15)$ & $3(60)$ & 0.02 \\
\hline Child-Pugh class, $\mathrm{n}(\%)(\mathrm{A}, \mathrm{B})$ & $41(91), 4(9)$ & $36(90), 4(10)$ & $5(100), 0$ & 0.76 \\
\hline ALBI grade, $\mathrm{n}(\%)(1,2,3)$ & 21 (47), 21 (47), 3 (8) & $19(48), 18(45), 3$ (7) & $2(40), 3(60), 0$ & 0.90 \\
\hline TNM stage, n (\%) (II, III, IVA, IVB) & $4(9), 1(2), 9(20), 31(69)$ & $3(8), 1(3), 9(23), 27(66)$ & $1(20), 0,0,4(80)$ & 0.79 \\
\hline
\end{tabular}

BMI: Body mass index; ECOG-PS: Eastern Cooperative Oncology Group performance status; eGFR: estimated glomerular filtration rate; HBV: hepatitis B virus; HCV: hepatitis C virus; ACE inhibitor: angiotensin converting enzyme inhibitor; ARB: angiotensin II receptor blocker; NSAID: non-steroidal anti-inflammatory drug; NRP, nephrotic-range proteinuria; AFP: alpha-fetoprotein; ALBI grade: albumin-bilirubin grade; TNM grade: tumor node metastasis stage.

Study Group of Japan (10). Other inclusion criteria were age $\geq 18$ years and patients who were receiving lenvatinib between September 1, 2018 and December 31, 2019. Similarly, we restricted the analytic sample to patients with at least 1 month of follow-up from the initiation of lenvatinib. Lenvatinib was administered at $12 \mathrm{mg} /$ day if the body weight was $<60 \mathrm{~kg}$ or $8 \mathrm{mg} /$ day if the body weight was $\geq 60$ $\mathrm{kg}$. Dose adjustments followed by reductions to $8 \mathrm{mg} / \mathrm{day}, 4 \mathrm{mg} /$ day, or $4 \mathrm{mg}$ every other day were allowed when a patient experienced an adverse event of grade 3 or above, and lenvatinib was resumed when adverse effects improved to grade 1 or 2 . Adverse effects were graded based on the Common Terminology Criteria for Adverse Effects version 4.0 (11). This study was conducted in compliance with the Declaration of Helsinki and approved by the Institutional Review Board of Teine Keijinkai Medical Center (approval number 2-019169$00)$. Written informed consent was obtained from all patients at the start of the treatment but was not required for the analyses because this was a retrospective study. This study was conducted and reported according to the Strengthening the Reporting of Observational Studies in Epidemiology (STROBE) guidelines (12).

Exposures. The primary exposure of interest was NRP, which was defined as the first record of urine protein to creatinine ratio of $>3$ $\mathrm{g} / \mathrm{gCr}$ or a urine protein dipstick indicator of $\geq 4+$ after lenvatinib initiation based on the Kidney Disease: Improving Global Outcomes clinical practice guidelines for glomerulonephritis (13). After the initiation of lenvatinib, proteinuria was measured at every scheduled visit of each patient.

Outcome. The primary outcome evaluated was the overall survival time, which was defined as the time from the initiation of lenvatinib treatment to the date of death or the patient's last follow-up.

Covariates. We collected data on covariates by reviewing patient medical records. Patient characteristics included age, sex, body mass index, comorbidities (hypertension, diabetes mellitus, heart failure, and chronic kidney disease), ECOG-PS score, (14) causes of HCC (hepatitis $\mathrm{C}$, hepatitis $\mathrm{B}$, and other causes), macrovascular invasion, extrahepatic spread, and prescribed medications (non-steroidal anti-inflammatory drugs and RASi). Previous treatment outcomes, including sorafenib and transcatheter arterial chemoembolization, were also recorded. Laboratory data were based on the first measurement after 1 month of lenvatinib initiation and included total bilirubin levels, serum albumin, prothrombin time using the international normalized ratio, alphafetoprotein, $\mathrm{sCr}$, estimated glomerular filtration rate (eGFR), which was calculated using the three-variable equation for Japanese individuals (15), and pre-existing grade 1 proteinuria, which was defined as a urine protein to creatinine ratio of $>0.3 \mathrm{~g} / \mathrm{gCr}$ or urine protein dipstick of $\geq 1+$ (11). Hypertension was defined as a systolic blood pressure $\geq 140$ $\mathrm{mmHg}$, a diastolic blood pressure $\geq 90 \mathrm{mmHg}$, or antihypertensive drug use. Diabetes mellitus was defined as a hemoglobin A1c level of $\geq 6.5 \%$ 
Table II. Clinical factors affecting all-cause mortality.

\begin{tabular}{|c|c|c|c|c|c|c|}
\hline & \multicolumn{3}{|c|}{ Univariable } & \multicolumn{3}{|c|}{ Multivariable } \\
\hline & Hazard ratio & $95 \% \mathrm{CI}$ & $p$-Value & Hazard ratio & $95 \% \mathrm{CI}$ & $p$-Value \\
\hline NRP & 0.55 & $0.12-2.52$ & 0.44 & 0.86 & $0.15-4.93$ & 0.86 \\
\hline Age & 1.01 & $0.97-1.05$ & 0.66 & 1.01 & $0.95-1.07$ & 0.83 \\
\hline CKD & 1.04 & $0.32-3.39$ & 0.95 & 0.68 & $0.12-3.74$ & 0.66 \\
\hline Child-Pugh class & 1.30 & $0.16-10.6$ & 0.81 & 0.82 & $0.09-7.62$ & 0.87 \\
\hline TNM stage & 0.83 & $0.46-1.52$ & 0.55 & 0.78 & $0.41-1.48$ & 0.45 \\
\hline ECOG-PS & 1.57 & $0.89-2.79$ & 0.12 & 1.63 & $0.79-3.39$ & 0.19 \\
\hline
\end{tabular}

CI: Confidence interval; CKD: chronic kidney disease; ECOG-PS: Eastern Cooperative Oncology Group performance status; NRP: nephrotic-range proteinuria; TNM stage: tumor, node, metastasis stage.

(16) or the use of anti-diabetic drugs. CKD was defined as an eGFR $<60 \mathrm{ml} / \mathrm{min} / 1.73 \mathrm{~m}^{2}$ for $>3$ months before the start of lenvatinib. We evaluated hepatic reserves using the Child-Pugh class and ALBI grade (17). HCC was staged according to the Liver Cancer Study Group of Japan (10). Similarly, other lenvatinib-related adverse events, including fatigue, hand-foot skin reaction, appetite loss, thrombocytopenia, diarrhea, and rash, were recorded according to the Common Terminology Criteria for Adverse Effects version 4.0 (11).

Statistical analysis. All analyses were performed using STATA version 15.1 (Stata Corp LLC, College Station, TX, USA). We compared baseline characteristics between patients who developed NRP and those who did not develop NRP using the chi-squared test for categorical variables, Student's $t$-test for normally distributed continuous variables, and Wilcoxon rank-sum test for non-normally distributed continuous variables. Kaplan-Meier curve analyses, with the log-rank test, were performed to compare the overall survival between patients who developed NRP and those who did not. Additionally, univariable and multivariable Cox proportional hazard analyses were performed to estimate the hazard ratio and $95 \%$ confidence interval (CI) for all-cause mortality. Independent variables analyzed by Cox proportional hazard models included NRP, age, chronic kidney disease, Child-Pugh class, cancer stage, (10) and ECOG-PS, (14) and were selected based on previous studies $(6,18$ $21)$ and our clinical experience. All variables used in the univariate analyses were incorporated into the multivariable analysis as independent variables. Additionally, we performed univariable and multivariable logistic regression analyses to identify the risk factors for NRP. Odds ratios and corresponding 95\%CIs were calculated. Based on previous studies $(18,19,21)$, we selected pre-existing proteinuria, age, RASi, CKD, hypertension, and diabetes mellitus as independent variables in the logistic regression analyses. All variables used in the univariable analyses were incorporated into the multivariable analysis as independent variables. A two-sided $p$-value $<0.05$ was considered statistically significant. Due to the limited sample size, subgroup and sensitivity analyses were not performed.

\section{Results}

Baseline characteristics of the study population. In total, 46 consecutive patients who were administered lenvatinib between September 1, 2018 and December 31, 2019 were potentially eligible for the study, and 45 patients were included in our analyses after excluding one patient with less than 1 month of follow-up data. The median age was 66 [interquartile range $(\mathrm{IQR})=55-72]$ years, $56 \%$ of the patients were women, and $20 \%$ had pre-existing proteinuria. Fourteen patients $(31 \%)$ received sorafenib prior to lenvatinib treatment. Patients who developed NRP were more likely to have heart failure and preexisting proteinuria and had higher serum creatinine $(\mathrm{sCr})$ levels than patients who did not develop NRP. Other parameters, including the Child-Pugh class, the albuminbilirubin (ALBI) grade, and the TNM grade did not differ significantly between the two groups (Table I).

Overall survival. During a median follow-up of 1 year (IQR=0.6-2.6 years), 25 patients died (56\%). The KaplanMeier survival analysis between the NRP and the non-NRP group showed that overall survival was not statistically different between the two groups (log-rank test, $p=0.4329$ ) (Figure 1). In univariable Cox proportional hazard analyses, NRP was not associated with overall survival. A similar result was observed in the multivariable analysis, which was adjusted for age, chronic kidney disease (CKD), Child-Pugh class, TNM stage, and Eastern Cooperative Oncology Group Performance Status (ECOG-PS) (Table II).

Risk factors for NRP with lenvatinib. NRP occurred in five patients $(11 \%)$ at a median of 5 months (IQR=3.1-12.0 months) after the initiation of lenvatinib; therefore, lenvatinib was discontinued in all patients with NRP. Univariable analyses revealed that pre-existing proteinuria was associated with a higher risk of NRP development. However, the multivariable analysis failed to demonstrate any significant clinical factors associated with NRP (Table III).

Other adverse events. Twenty-three patients (50\%) developed adverse events other than NRP. Fatigue was the most common adverse event (10 events), followed by hand-foot skin reactions (6 events), and appetite loss (2 events; Table IV). 


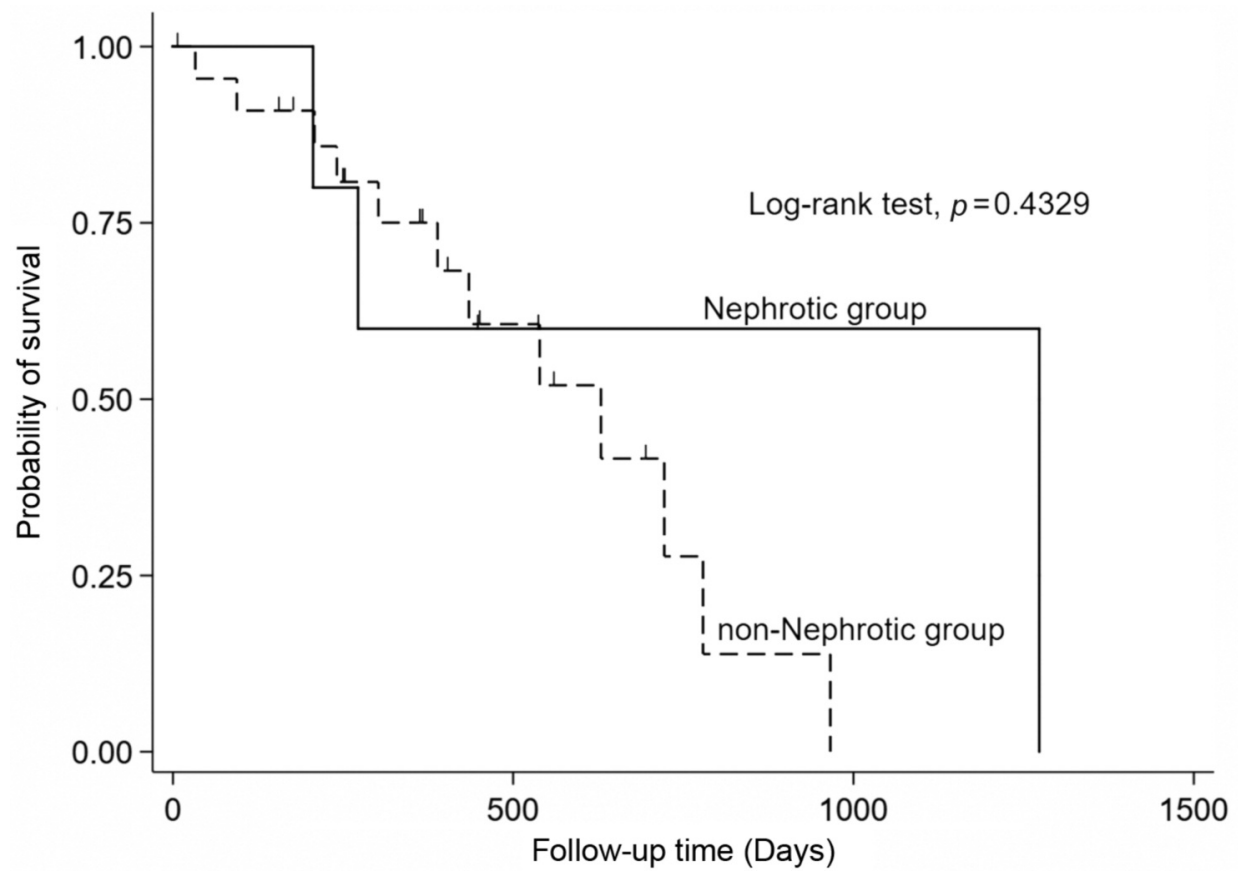

Figure 1. Kaplan-Meier curves showing mortality in the nephrotic and the non-nephrotic groups.

Table III. Clinical factors associated with the incidence of nephrotic-range proteinuria.

\begin{tabular}{|c|c|c|c|c|c|c|}
\hline & \multicolumn{3}{|c|}{ Univariable } & \multicolumn{3}{|c|}{ Multivariable } \\
\hline & Odds ratio & $95 \% \mathrm{CI}$ & $p$-Value & Odds ratio & $95 \% \mathrm{CI}$ & $p$-Value \\
\hline Pre-existing proteinuria & 8.50 & $1.16-62.1$ & 0.04 & 8.55 & $0.67-109$ & 0.10 \\
\hline Age & 1.06 & $0.96-1.17$ & 0.24 & 1.14 & $0.95-1.38$ & 0.25 \\
\hline RAS inhibitors & 3.27 & $0.34-31.9$ & 0.31 & 0.90 & $0.65-17.6$ & 0.94 \\
\hline CKD & 1.00 & $0.15-6.67$ & 1.00 & 0.40 & $0.03-4.92$ & 0.47 \\
\hline Hypertension & 1.00 & $0.11-6.12$ & 1.00 & 1.00 & $0.17-3.99$ & 0.99 \\
\hline Diabetes mellitus & 0.52 & $0.05-5.12$ & 0.57 & 0.19 & $0.10-3.76$ & 0.28 \\
\hline
\end{tabular}

CI: Confidence intervals; RAS inhibitors: renin-angiotensin system inhibitors; CKD: chronic kidney disease.

\section{Discussion}

In this single-centre observational study of Japanese patients with HCC treated with lenvatinib, we showed that NRP development after lenvatinib initiation was not significantly associated with overall survival. In unadjusted logistic regression analysis, patients with pre-existing proteinuria had an 8.5-times higher risk of NRP development during treatment; however, this association was not observed after adjusting for age, renin-angiotensin system inhibitors (RASi), CKD, and diabetes mellitus.

Our study is unique because, to the best of our knowledge, this is the first study assessing the association between NRP development during treatment and overall survival in patients with $\mathrm{HCC}$ receiving lenvatinib. Indeed, a recent meta-analysis revealed that lenvatinib was associated with a 4.1-times increased risk of developing high-grade proteinuria compared to other vascular endothelial growth factor receptor-tyrosine kinase inhibitors; however, the effect of lenvatinib-associated proteinuria on overall survival was not evaluated (18). Additionally, previous studies have reported several prognostic factors for progression-free survival, including age, tumor stage, ChildPugh class, and performance status $(6,19,20)$. However, in the present study, none of these factors was significantly associated with overall survival. Thus, to elucidate the 
Table IV. Lenvatinib-associated adverse events.

\begin{tabular}{lccc}
\hline & All (n=45) & $\begin{array}{c}\text { non-NRP } \\
(\mathrm{n}=40)\end{array}$ & $\begin{array}{c}\text { NRP } \\
(\mathrm{n}=5)\end{array}$ \\
\hline Any adverse event, n (\%) & $23(50)$ & $21(53)$ & $2(40)$ \\
Fatigue, n (\%) & $10(22)$ & $9(23)$ & $1(20)$ \\
Hand-foot skin reaction, n (\%) & $6(13)$ & $6(15)$ & 0 \\
Appetite loss, n (\%) & $2(4)$ & $2(5)$ & 0 \\
Thrombocytopenia, n (\%) & $1(2)$ & $1(3)$ & 0 \\
Diarrhea, n (\%) & $1(2)$ & $1(3)$ & 0 \\
Rash, n (\%) & $1(2)$ & $1(3)$ & 0 \\
\hline
\end{tabular}

NRP: Nephrotic-range proteinuria.

association between NRP development during treatment and overall survival in HCC patients receiving lenvatinib, multicenter prospective studies with larger sample sizes are required in the future.

Our study showed that the rate of NRP development during treatment in patients with $\mathrm{HCC}$ receiving lenvatinib was $11 \%$, which was consistent with the results of previous studies (4, $7-9,20)$. A phase III randomized controlled trial showed that the incidence of grade 3-4 proteinuria associated with lenvatinib was $6 \%,(4)$ and other studies have demonstrated that the rate of grade 3-4 proteinuria ranged from 5 to $10 \%$ (79, 20). Pre-existing proteinuria, hypertension, diabetes mellitus, and CKD have been reported as potential predictors of proteinuria during treatment with vascular endothelial growth factor-targeted therapy $(19,21)$. Similarly, a significant association was not observed between pre-existing proteinuria and NRP development during treatment, after adjustment for clinical factors. This discrepancy may be due to the differences in the study populations and definitions of outcomes. Specifically, the aforementioned studies $(19,21)$ enrolled patients with metastatic renal cell carcinoma treated with sunitinib or pazopanib, whereas our study included patients with advanced HCC receiving lenvatinib.

The precise mechanism of proteinuria in patients treated with lenvatinib is yet to be elucidated; however, several potential mechanisms have been proposed $(18,22-26)$. One possibility is that arterial hypertension may cause an increase in intraglomerular pressure that worsens proteinuria $(18,22)$. However, this has been challenged by the results of a study using a mouse model, which demonstrated that podocyte damage induced by reduced expression of vascular endothelial growth factor existed before the development of hypertension (23). In our analyses, hypertension was not significantly associated with a higher risk of NRP development during treatment. Another possibility is that lenvatinib may cause a loss of selective glomerular permeability $(24,25)$. Finally, renal damage by lenvatinib may impair the repair process in the glomeruli, leading to glomerulosclerosis (26).
This study suggests that NRP should be recognized as one of the major adverse effects of lenvatinib. Monitoring for NRP during treatment is important as it can influence additional antitumor treatments in patients receiving lenvatinib. Moreover, the development of NRP during lenvatinib treatment may preclude treatment with other antitumor agents. Further studies to investigate the predictors of NRP in this population are warranted.

The present study had several limitations. First, this study did not have adequate power to detect all potentially significant differences because this was a single-centre study with a small sample size. Moreover, due to the small number of events, the rule of ten events per dependent variable was not strictly followed. Second, the observation period was relatively short, mainly because lenvatinib has only been approved to treat advanced HCC in Japan since March 2018. Third, the possibility of ascertainment bias exists because the timing of data collection during the observation period relied on the attending physicians, and it is possible that several cases of NRP were missed. Fourth, patients who had received sorafenib or underwent transcatheter arterial chemoembolization prior to lenvatinib administration were included in the study; therefore, the effect of lenvatinib on overall survival may have been underestimated. Fifth, the possibility of residual confounding exists because of the retrospective observational design of this study. Finally, our study population included a cohort of Japanese patients with advanced HCC; thus, the generalizability of our findings to other populations should be interpreted with caution.

In conclusion, this study suggests that NRP development after lenvatinib initiation does not alter the overall survival of patients with advanced HCC. Future large-scale studies are necessary to explore the effect of NRP development on the clinical outcomes of patients with advanced HCC receiving lenvatinib.

\section{Conflicts of Interest}

The Authors declare no competing interests in relation to this study.

\section{Authors' Contributions}

Research idea and study design: Y.S., T.M., A.I., H.T.; data acquisition: Y.S., K.A.; data analysis/interpretation: Y.S., T.M.; statistical analysis: Y.S.; supervision or mentorship: T.M., H.T. Each author contributed important intellectual content during manuscript drafting or revision and accepts accountability for the overall work by ensuring that questions pertaining to the accuracy or integrity of any portion of the work are appropriately investigated and resolved.

\section{Acknowledgements}

The Authors would like to thank Editage (www.editage.com) for English language editing. 


\section{References}

1 Bray F, Ferlay J, Soerjomataram I, Siegel RL, Torre LA and Jemal A: Global cancer statistics 2018: GLOBOCAN estimates of incidence and mortality worldwide for 36 cancers in 185 countries. CA Cancer J Clin 68(6): 394-424, 2018. PMID: 30207593. DOI: $10.3322 /$ caac. 21492

2 Llovet JM, Ricci S, Mazzaferro V, Hilgard P, Gane E, Blanc JF, de Oliveira AC, Santoro A, Raoul JL, Forner A, Schwartz M, Porta C, Zeuzem S, Bolondi L, Greten TF, Galle PR, Seitz JF, Borbath I, Häussinger D, Giannaris T, Shan M, Moscovici M, Voliotis D, Bruix J and SHARP Investigators Study Group: Sorafenib in advanced hepatocellular carcinoma. N Engl J Med 359(4): 378-390, 2008. PMID: 18650514. DOI: 10.1056/ NEJMoa0708857

3 Cheng AL, Kang YK, Chen Z, Tsao CJ, Qin S, Kim JS, Luo R, Feng J, Ye S, Yang TS, Xu J, Sun Y, Liang H, Liu J, Wang J, Tak WY, Pan H, Burock K, Zou J, Voliotis D and Guan Z: Efficacy and safety of sorafenib in patients in the Asia-Pacific region with advanced hepatocellular carcinoma: a phase III randomised, double-blind, placebo-controlled trial. Lancet Oncol 10(1): 25-34, 2009. PMID: 19095497. DOI: 10.1016/S14702045(08)70285-7

4 Kudo M, Finn RS, Qin S, Han KH, Ikeda K, Piscaglia F, Baron A, Park JW, Han G, Jassem J, Blanc JF, Vogel A, Komov D, Evans TRJ, Lopez C, Dutcus C, Guo M, Saito K, Kraljevic S, Tamai T, Ren M and Cheng AL: Lenvatinib versus sorafenib in first-line treatment of patients with unresectable hepatocellular carcinoma: a randomised phase 3 non-inferiority trial. Lancet 391(10126): 1163-1173, 2018. PMID: 29433850. DOI: 10.1016/S0140-6736(18)30207-1

5 Matsui J, Yamamoto Y, Funahashi Y, Tsuruoka A, Watanabe T, Wakabayashi T, Uenaka T and Asada M: E7080, a novel inhibitor that targets multiple kinases, has potent antitumor activities against stem cell factor producing human small cell lung cancer H146, based on angiogenesis inhibition. Int J Cancer 122(3): 664-671, 2008. PMID: 17943726. DOI: 10.1002/ ijc. 23131

6 Haddad RI, Schlumberger M, Wirth LJ, Sherman EJ, Shah MH, Robinson B, Dutcus CE, Teng A, Gianoukakis AG and Sherman SI: Incidence and timing of common adverse events in Lenvatinib-treated patients from the SELECT trial and their association with survival outcomes. Endocrine 56(1): 121-128, 2017. PMID: 28155175. DOI: 10.1007/s12020-017-1233-5

7 Ohki T, Sato K, Kondo M, Goto E, Sato T, Kondo Y, Akamatsu M, Sato S, Yoshida H, Koike Y and Obi S: Impact of adverse events on the progression-free survival of patients with advanced hepatocellular carcinoma treated with lenvatinib: A multicenter retrospective study. Drugs Real World Outcomes 7(2): 141-149, 2020. PMID: 32048238. DOI: 10.1007/s40801-020-00179-7

8 Nakano M, Kuromatsu R, Niizeki T, Okamura S, Iwamoto H, Shimose S, Shirono T, Noda Y, Kamachi N, Koga H, Torimura $\mathrm{T}$ and Kurume Liver Cancer Study Group of Japan: Primary treatment with molecular-targeted agents for hepatocellular carcinoma: A propensity score-matching analysis. Hepatol Commun 4(8): 1218-1228, 2020. PMID: 32766480. DOI: 10.1002/hep4.1535

9 Hiraoka A, Kumada T, Kariyama K, Takaguchi K, Itobayashi E, Shimada N, Tajiri K, Tsuji K, Ishikawa T, Ochi H, Hirooka M, Tsutsui A, Shibata H, Tada T, Toyoda H, Nouso K, Joko K,
Hiasa Y, Michitaka K and Real-life Practice Experts for HCC (RELPEC) Study Group and the HCC 48 Group (hepatocellular carcinoma experts from 48 clinics in Japan): Therapeutic potential of lenvatinib for unresectable hepatocellular carcinoma in clinical practice: Multicenter analysis. Hepatol Res 49(1): 111-117, 2019. PMID: 30144256. DOI: 10.1111/hepr.13243

10 The Liver Cancer Study Group of Japan. The general rules for the clinical and pathological study of primary liver cancer $6^{\text {th }}$ edition; Kanehara, 2015.

11 National Institute of Health. Common terminology criteria for adverse events v. 4.0 (CTCAE). Bethesda: NIH, 2009.

12 von Elm E, Altman DG, Egger M, Pocock SJ, Gøtzsche PC, Vandenbroucke JP and STROBE Initiative: The Strengthening the Reporting of Observational Studies in Epidemiology (STROBE) statement: guidelines for reporting observational studies. J Clin Epidemiol 61(4): 344-349, 2008. PMID: 18313558. DOI: $10.1016 /$ j.jclinepi.2007.11.008

13 von Elm E, Altman DG, Egger M, Pocock SJ, Gøtzsche PC, Vandenbroucke JP and STROBE Initiative.: The Strengthening the Reporting of Observational Studies in Epidemiology (STROBE) statement: guidelines for reporting observational studies. J Clin Epidemiol 61(4): 344-349, 2008. PMID: 18313558. DOI: $10.1016 /$ j.jclinepi.2007.11.008

14 Oken MM, Creech RH, Tormey DC, Horton J, Davis TE, McFadden ET and Carbone PP: Toxicity and response criteria of the Eastern Cooperative Oncology Group. Am J Clin Oncol 5(6): 649-655, 1982. PMID: 7165009.

15 Matsuo S, Imai E, Horio M, Yasuda Y, Tomita K, Nitta K, Yamagata K, Tomino Y, Yokoyama H, Hishida A and Collaborators developing the Japanese equation for estimated GFR.: Revised equations for estimated GFR from serum creatinine in Japan. Am J Kidney Dis 53(6): 982-992, 2009. PMID: 19339088. DOI: 10.1053/j.ajkd.2008.12.034

16 American Diabetes Association.: Executive summary: Standards of medical care in diabetes-2014. Diabetes Care 37 Suppl 1: S513, 2014. PMID: 24357214. DOI: 10.2337/dc14-S005

17 Johnson PJ, Berhane S, Kagebayashi C, Satomura S, Teng M, Reeves HL, O'Beirne J, Fox R, Skowronska A, Palmer D, Yeo W, Mo F, Lai P, Iñarrairaegui M, Chan SL, Sangro B, Miksad R, Tada T, Kumada $\mathrm{T}$ and Toyoda H: Assessment of liver function in patients with hepatocellular carcinoma: a new evidence-based approach-the ALBI grade. J Clin Oncol 33(6): 550-558, 2015. PMID: 25512453. DOI: 10.1200/JCO.2014.57.9151

18 Zhang W, Feng LJ, Teng F, Li YH, Zhang X and Ran YG: Incidence and risk of proteinuria associated with newly approved vascular endothelial growth factor receptor tyrosine kinase inhibitors in cancer patients: an up-to-date meta-analysis of randomized controlled trials. Expert Rev Clin Pharmacol 13(3): 311-320, 2020. PMID: 32105149. DOI: 10.1080/17512433. 2020.1734450

19 Baek SH, Kim H, Lee J, Kim DK, Oh KH, Kim YS, Han JS, Kim TM, Lee SH and Joo KW: Renal adverse effects of sunitinib and its clinical significance: a single-center experience in Korea. Korean J Intern Med 29(1): 40-48, 2014. PMID: 24574832. DOI: $10.3904 / \mathrm{kjim} .2014 .29 .1 .40$

20 Wang DX, Yang X, Lin JZ, Bai Y, Long JY, Yang XB, Seery S and Zhao HT: Efficacy and safety of lenvatinib for patients with advanced hepatocellular carcinoma: A retrospective, real-world study conducted in China. World J Gastroenterol 26(30): 44654478, 2020. PMID: 32874058. DOI: 10.3748/wjg.v26.i30.4465 
21 Sorich MJ, Rowland A, Kichenadasse G, Woodman RJ and Mangoni AA: Risk factors of proteinuria in renal cell carcinoma patients treated with VEGF inhibitors: a secondary analysis of pooled clinical trial data. Br J Cancer 114(12): 1313-1317, 2016. PMID: 27228299. DOI: 10.1038/bjc.2016.147

22 Cosmai L, Gallieni M, Liguigli W and Porta C: Renal toxicity of anticancer agents targeting vascular endothelial growth factor (VEGF) and its receptors (VEGFRs). J Nephrol 30(2): 171-180, 2017. PMID: 27154025. DOI: 10.1007/s40620-016-0311-8

23 Eremina V, Jefferson JA, Kowalewska J, Hochster H, Haas M, Weisstuch J, Richardson C, Kopp JB, Kabir MG, Backx PH, Gerber HP, Ferrara N, Barisoni L, Alpers CE and Quaggin SE: VEGF inhibition and renal thrombotic microangiopathy. N Engl J Med 358(11): 1129-1136, 2008. PMID: 18337603. DOI: 10.1056/NEJMoa0707330

24 Izzedine H, Mangier M, Ory V, Zhang SY, Sendeyo K, Bouachi K, Audard V, Péchoux C, Soria JC, Massard C, Bahleda R, Bourry E, Khayat D, Baumelou A, Lang P, Ollero M, Pawlak A and Sahali D: Expression patterns of RelA and c-mip are associated with different glomerular diseases following antiVEGF therapy. Kidney Int 85(2): 457-470, 2014. PMID: 24067439. DOI: $10.1038 / \mathrm{ki} .2013 .344$
25 Hara A, Wada T, Furuichi K, Sakai N, Kawachi H, Shimizu F, Shibuya M, Matsushima K, Yokoyama H, Egashira K and Kaneko S: Blockade of VEGF accelerates proteinuria, via decrease in nephrin expression in rat crescentic glomerulonephritis. Kidney Int 69(11): 1986-1995, 2006. PMID: 16641924. DOI: 10.1038/sj.ki.5000439

26 Kamba T, Tam BYY, Hashizume H, Haskell A, Sennino B, Mancuso MR, Norberg SM, O'Brien SM, Davis RB, Gowen LC, Andersen KD, Thurston G, Joho S, Springer ML, Kuo CJ and McDonold DM: VEGF-dependent plasticity of fenestrated capillaries in the normal adult microvasculature. Am J Physiol Heart Circ Physiol 290(2): 560-576, 2006. PMID: 16172168. DOI: 10.1152/ajphert.00133.2005
Received January 25, 2021

Revised February 6, 2021

Accepted February 12, 2021 